\title{
Inflammatory abdominal aortic aneurysm presenting as bilateral hydroureteronephrosis: A case report and review of literature
}

\author{
Andrea Benedetto Galosi ${ }^{1}$, Carlo Grilli Cicilioni ${ }^{2}$, Giulia Sbrollini ${ }^{1}$, Andrea Angelini ${ }^{2}$, \\ Guevar Maselli ${ }^{1}$, Luciano Carbonari ${ }^{2}$ \\ ${ }^{1}$ UOC Urologia, Ospedale "Augusto Murri", Fermo, Area Vasta n 4, ASUR Marche; \\ ${ }^{2}$ UOC Chirurgia Vascolare, Ospedali Riuniti, Ancona, Italy.
}

\begin{abstract}
Summary We report a case of Inflammatory
Abdominal Aortic Aneurysm (IAAA)

producing bilateral hydro-ureteronephrosis. A 74-year-old patient presented to urologist office for bilateral hydronephrosis detected by kidney and bladder ultrasound (US). Patient reported lower urinary tract symptoms and inconstant and slight low back pain irradiated to inguinal region dating 3 weeks. Renal function, urine analysis and abdominal examination were normal. However the repeated ultrasound in the urologist office revealed abdominal aortic aneurism extended to iliac vessels. The patient was sent directly to vascular surgery unit where contrast computerized tomography (CT) and successful surgical repair were done. Final diagnosis was IAAA. The post-operative course was uneventful. Renal function was regular and the hydronephrosis reduced spontaneously under monitoring by CT and US. We review diagnosis and management of hydronephrosis that is sometimes linked to IAAA rather than standard AAA. Abdominal ultrasound is mandatory in any bilateral hydronephrosis and it could save lives.
\end{abstract}

KEY WORDS: Hydronephrosis; Inflammatory abdominal aortic aneurysm; Ultrasound; Kidney; Diagnosis.

Submitted 3 October 2014; Accepted 31 October 2014

\section{INTRODUCTION}

Abdominal aortic aneurysms are rare causes of ureteric obstruction. However Inflammatory Abdominal Aortic Aneurysm (IAAA) may be associated to ureteral obstruction in relation to peri-aortic inflammation in $30-20 \%$ of cases $(1,2)$. We report a case of IAAA producing bilateral hydroureteronephrosis in a patient with normal renal function, urinary symptoms and mild abdominal pain not requiring analgesics.

\section{Case report}

A 74-year-old patient presented to the urologist office for bilateral hydronephrosis detected by kidney and bladder ultrasound in ultrasound clinic, that was prescribed by General Physician. Patient reported mild and inconstant low back pain irradiated to inguinal region dating 3 weeks associated to lower urinary tract symptoms. Then the patients was referred to the urologist to evaluate hydronephrosis and symptoms. He had visceral obesity, hypertension and hyperuricemia. Renal function and urine analysis were normal. Abdominal examination was negative. The former ultrasound (US) was restricted to urinary tract only. At abdominal US done in the urologist office any stone or solid mass was detected in the kidney or in the bladder, but US showed a thick and hypoechoic wall (diameter $2 \mathrm{~cm}$ ) located around the aorta and iliac vessels. The US diagnosis was suspected fractured abdominal aortic aneurism extended to iliac vessels (Figure 1).

The patient was sent directly to emergency department, where it was referred to vascular surgery unit where contrast chest and abdominal computerized tomography (CT) was carried out and subsequent surgery was performed. Severe hypertension was the reason for emergency surgery. Successful repair with an open approach using a bifurcated dacron graft was made. Final diagnosis was IAAA. Immunoglobulin G4-related systemic disease was ruled out by pathologist on the surgical specimen of the aortic wall. The post-operative course was uneventful, the renal function was in the normal range and the hydronephrosis was monitored by CT and US. Hydronephrosis spontaneously reduced and the patien was followed for after 8 months to detect complete regression or retroperitoneal fibrosis.

\section{Discussion}

IAAAs represent from 3 to $10 \%$ of all abdominal aortic aneurysms and are associated with peri-aortic inflammation $(2,3)$. The most common clinical features of these aneurysms are represented by symptoms, such as abdominal or back pain, obstructive uropathy ( $1 / 4$ of cases) and by an elevated VES, PCR. However isolated hip pain associated with tender bilateral testicular swellings may be the 


\section{Figure 1.}

Abdominal contrast $C T(A+B)$ showing bilateral hydronephrosis, periaortic soft and tick tissue (I) with inflammatory tissue, that appear as hypoechoic at the US (D). Echocolor-doppler (C) showing the aorta and the inferior mesenteric artery (arrow) inside the thick inflammatory wall of the aneurism.

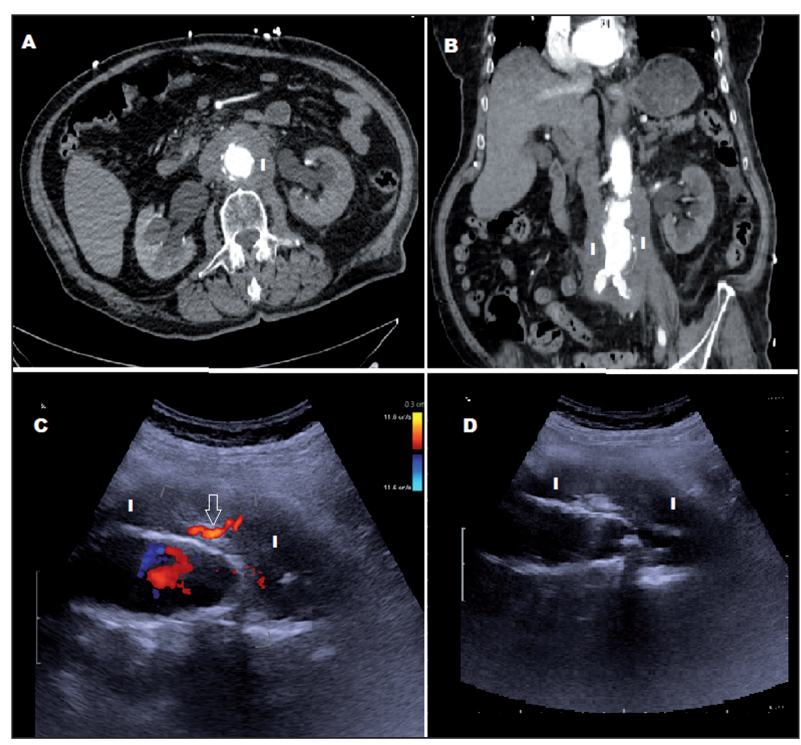

presentation symptoms. Entrapment of near-standing organs can develop with compression of the ureter and the duodenum and consequent hydroureteronephrosis and bowel obstruction (4). Preoperative diagnosis is possible by abdominal US. CT allows a specific diagnosis by the typical image of soft tissue surrounding the aortic wall enhanced with contrast administration ("enhancing periaortic soft-tissue mantle sign") and demonstrates ureter entrapment, mural aortic thrombus and wall calcification (5). In patients with acute renal failure or even obstructive anuria the diagnosis can be suspected by non-contrast CT and Echo-Doppler US and the diagnosis established by contrast CT. Pre-operative ureteral stenting is indicated in case of severe renal failure.

Evolution of peri-ureteral or retroperitoneal fibrosis after surgery is still debated because some studies have reported complete regression of inflammation and others partial regression or persistence of fibrotic process. Often surgical treatment is inadequate to control retroperitorenal fibrosis and so the surgeon has to use perioperating pharmacolocical therapy and/or ureteral stenting. Despite the similarity of Infected and Inflammatory AAA, the infected one generally shows a more rapid change in clinical condition, leading to a fatal outcome; in addition, delayed diagnosis and misuse of corticosteroid or immunosuppressing drugs may lead to uncontrolled growth of microorganisms $(4,5)$. It is mandatory that detection of aortic aneurysm is followed by accurate differential diagnosis among aortic aneurysm, infected and inflammatory aortic aneurysm. Differential diagnosis between Infected and Inflammatory sometimes may not be easy for the following reasons: (1) symptoms, such as abdominal and/or back pain and fever, and blood test abnormalities, such as elevated C-reactive protein and enhanced erythrocyte sedimentation rate, are common in infected aortic aneurysm, but they are not found infrequently also in inflammatory aortic aneurysm; (2) some inflammatory AAA are immunoglobulin G4-related, but not all of them; (3) the prevalence of $\operatorname{IgG} 4$ positivity in infected/inflammatory aortic aneurysm has not been well investigated $(6,7)$. Furthermore is recommended to rule out IgG4-related systemic disease in order to estabilish the usefulness of steroid therapy and proper follow-up.

\section{Conclusion}

Abdominal Ultrasonography is the first step in the diagnostic pathway of hydronephrosis. Potentially life-threatening diagnoses including AAA may mimic renal colic or inflammatory-AAA give hydronephrosis (mono or bilateral) and must be ruled out. Monolateral or bilateral Hydronephrosis can be associated more frequently to IAAA than non-inflammatory AAA. In IAAA, the IgG4 systemic disease should be ruled out.

\section{REFERENCES}

1. Sánchez R, Arroyo A, Gesto R, et al. Obstructive ARF caused by an inflammatory abdominal aortic aneurysm. Am J Kidney Dis. 2003; 41:E9.

2. Ruiz de la Illa S, Vega Manrique R, Lacasa Viscasillas I, et al. Inflammatory aortic aneurysm and its relationship to urological disease: a case report and a literature review. Actas Urol Esp. 2009; 33:1024-7.

3. Carter MR, Green BR. Renal calculi: emergency department diagnosis and treatment. Emerg Med Pract. 2011; 13:1-17

4. Rubini P, Bonati L, Parolari A, Spirito R. Inflammatory abdominal aortic aneurysms. Minerva Chir. 2001; 56:287-98.

5. Bajardi G, Pecoraro F, Mirabella D, Bellisi MG. Inflammatory abdominal aortic aneurysm. Ann Ital Chir. 2009; 80:171-6.

6. Kasashima S, Zen Y. IgG4-related inflammatory abdominal aortic aneurysm. Curr Opin Rheumatol. 2011; 23:18-23.

7. Mazzucchelli R, Racchini S, Barbisan F, et al. IgG4-related Sclerosing Disease: An Emerging Entity Frequently Misdiagnosed. Anal Quant Cytol Histol 2013; 35:189-196.

\section{Correspondence}

Andrea B. Galosi, MD (Corresponding Author)

galosiab@yahoo.it

Giulia Sbrollini, MD, Resident of Urology

Guevar Maselli, MD

Division of Urology, Dept. of Surgery, "Augusto Murri" General Hospital, Area Vasta 4, Az. Sanitaria Unica Regione Marche

63900 Fermo (FM), Italy

Carlo Grilli Cicilioni, MD

grillicicilioni@tiscali.it

Andrea Angelini, MD

angelini@fastwebnet.it

Luciano Carbonari, MD

luciano.carbonari@ospedaliriuniti.marche.it

UOC Chirurgia Vascolare, Ospedali Riuniti, Ancona, Italy 